\title{
Anomalous Magnetic Excitations of Cooperative Tetrahedral Spin Clusters
}

Prsa, K.; Rønnow, H.M.; Zaharko, O.; Christensen, Niels Bech; Jensen, J.; Chang, J.; Streule, S.; Jimenez-Ruiz, M.; Berger, H.; Prester, M.

Total number of authors:

11

Published in:

Physical Review Letters

Link to article, DOI:

10.1103/PhysRevLett.102.177202

Publication date:

2009

Document Version

Publisher's PDF, also known as Version of record

Link back to DTU Orbit

Citation $(A P A)$ :

Prsa, K., Rønnow, H. M., Zaharko, O., Christensen, N. B., Jensen, J., Chang, J., Streule, S., Jimenez-Ruiz, M., Berger, H., Prester, M., \& Mesot, J. (2009). Anomalous Magnetic Excitations of Cooperative Tetrahedral Spin Clusters. Physical Review Letters, 102(17), 177202. https://doi.org/10.1103/PhysRevLett.102.177202

\section{General rights}

Copyright and moral rights for the publications made accessible in the public portal are retained by the authors and/or other copyright owners and it is a condition of accessing publications that users recognise and abide by the legal requirements associated with these rights.

- Users may download and print one copy of any publication from the public portal for the purpose of private study or research.

- You may not further distribute the material or use it for any profit-making activity or commercial gain

- You may freely distribute the URL identifying the publication in the public portal 


\title{
Anomalous Magnetic Excitations of Cooperative Tetrahedral Spin Clusters
}

\author{
K. Prša, ${ }^{1, *}$ H. M. Rønnow, ${ }^{2}$ O. Zaharko, ${ }^{1}$ N. B. Christensen,,${ }^{1,3,4}$ J. Jensen,,${ }^{5}$ J. Chang, ${ }^{1}$ S. Streule, ${ }^{1}$ M. Jiménez-Ruiz, ${ }^{6}$ \\ H. Berger, ${ }^{7}$ M. Prester, ${ }^{8}$ and J. Mesot ${ }^{1,7}$ \\ ${ }^{1}$ Laboratory for Neutron Scattering, ETH Zürich and Paul Scherrer Institute, CH-5232 Villigen PSI, Switzerland \\ ${ }^{2}$ Laboratory for Quantum Magnetism, Ecole Polytechnique Fédérale de Lausanne, CH-1015 Lausanne, Switzerland \\ ${ }^{3}$ Materials Research Division, Ris $\phi$ DTU, Technical University of Denmark, DK-4000 Roskilde, Denmark \\ ${ }^{4}$ Nano-Science Center, Niels Bohr Institute, University of Copenhagen, DK-2100 Copenhagen, Denmark \\ ${ }^{5}$ Niels Bohr Institute, Universitetsparken 5, 2100 Copenhagen, Denmark \\ ${ }^{6}$ Institut Laue-Langevin, BP 156, 38042, Grenoble Cedex, France \\ ${ }^{7}$ Institut de Physique de la Matière Complexe, Ecole Polytechnique Fédérale de Lausanne, CH-1015 Lausanne, Switzerland \\ ${ }^{8}$ Institute of Physics, POB 304, HR-10 000, Zagreb, Croatia
}

(Received 28 October 2008; published 28 April 2009)

\begin{abstract}
An inelastic neutron scattering study of $\mathrm{Cu}_{2} \mathrm{Te}_{2} \mathrm{O}_{5} X_{2}(X=\mathrm{Cl}, \mathrm{Br})$ shows strong dispersive modes with large energy gaps persisting far above $T_{N}$, notably in $\mathrm{Cu}_{2} \mathrm{Te}_{2} \mathrm{O}_{5} \mathrm{Br}_{2}$. The anomalous features: a coexisting unusually weak Goldstone-like mode observed in $\mathrm{Cu}_{2} \mathrm{Te}_{2} \mathrm{O}_{5} \mathrm{Cl}_{2}$ and the size of the energy gaps cannot be explained by existing theories, such as our mean-field or random-phase approximation. We argue that our findings represent a new general type of behavior due to intercluster quantum fluctuations and call for development of a new theoretical approach.
\end{abstract}

DOI: 10.1103/PhysRevLett.102.177202

PACS numbers: 75.30.- m, 75.10.Jm, 78.70.Nx

An essential question in solid state physics is how local quantum states evolve into extended collective states. Contrary to the enormously complex spatially distributed electron problem, magnetic insulators offer an arena of clean, well defined spin models to pursue this evolution. An especially intriguing scenario occurs when the basic magnetic units consist themselves of several spinsclusters-which when isolated exhibit a nonmagnetic singlet ground state. Such systems oppose long-ranged magnetic order and, as we shall show, even when ordered retain features of their quantum ancestry.

Materials with isolated magnetic clusters have been studied intensively in the past because of their theoretical simplicity [1] - the magnetic ground state (GS) and excitations can usually be calculated analytically. The spectrum of an isolated $S=1 / 2$ tetrahedron with antiferromagnetic (AFM) exchange interactions consists of 16 discrete states, the GS being a singlet $S_{\text {tot }}=0$. When individual clusters, each with an internal level scheme and a singlet GS, are connected through intercluster interactions, the coupled system remains a singlet as long as interactions remain sufficiently weak. The discrete level scheme is expected to survive but the weak perturbative coupling gives rise to slight dispersion and possible lifting of degeneracies. In the opposite limit of comparable interand intracluster excitations the system may order where internal levels give way to regular dispersive collective excitations-spin waves.

The crystal structures of $\mathrm{Cu}_{2} \mathrm{Te}_{2} \mathrm{O}_{5} \mathrm{Cl}_{2}$ (CTC) and $\mathrm{Cu}_{2} \mathrm{Te}_{2} \mathrm{O}_{5} \mathrm{Br}_{2}$ (CTB) suggest $\mathrm{Cu}^{2+}$ tetrahedra as elementary magnetic units [2]. Susceptibility measurements yield intracluster interaction strengths of about $40 \mathrm{~K}$ in both materials [3]. CTC and CTB order magnetically at the relatively low $T_{N}=18$ and $11.4 \mathrm{~K}$, respectively. These compounds display a similar, possibly the most complex incommensurate (ICM) magnetic order yet solved for spin-1/2 systems [4-6], thus providing a solid benchmark for both experimental and theoretical methods. As for the excitations, inelastic neutron scattering (INS) on powders indicates a dispersive mode and a flat mode around $5.5 \mathrm{meV}$ for CTC and $6 \mathrm{meV}$ for CTB [7]. A recent Raman study [8] shows more details of the magnetic spectrum.

CTC and CTB are isostructural with only 7\% larger unit cell volume in CTB [2]. Yet, there are differences between the two materials. (i) The higher ordering temperature implies stronger intertetrahedral exchange interactions in CTC. (ii) In CTC the magnetic moment $\left(0.88 \mu_{B}\right)$ almost reaches the classical value of $1 \mu_{B}$ while it is strongly reduced $\left(0.4 \mu_{B}\right)$ in CTB [6]. (iii) INS measurements on powders have revealed different temperature dependencies of the magnetic features, which seem to persist high above the ordering temperature in CTB [7]. Here we report comprehensive single-crystal INS investigations, providing access to the full momentum, $\mathbf{Q}$, and energy, $\omega$, dependent spectrum $S(\mathbf{Q}, \omega)$.

Single crystals with reported magnetization behavior were grown by vapor transport [3]. We employed one large crystal of CTC with mass $7 \mathrm{~g}$, while for CTB five crystals of total mass $0.2 \mathrm{~g}$ were coaligned with an overall mosaicity of $2^{\circ}$. The samples were aligned in the $-k / 3, k, l$ and $(-k / 2, k, l)$ scattering planes to allow access to the magnetic ordering vectors $\mathbf{k}^{\prime}=(-0.150,0.422,1 / 2)$ and $\mathbf{k}^{\prime \prime}=(-0.172,0.356,1 / 2)$ for CTC and CTB, respectively 
[6]. The neutron scattering measurements were performed on the triple-axis spectrometers IN8 (thermal neutrons) and IN14 (cold neutrons) at ILL, Grenoble, France and TASP (cold neutrons) at SINQ, PSI, Switzerland.

Figure 1 shows intensity maps of the spectrum of CTC collected on IN8 at $T=2 \mathrm{~K} \ll T_{N}$, (a)-(b), and at $20 \mathrm{~K}$, (c)-(d), just above $T_{N}$. For all measured $\mathbf{Q}$, a mode is observed at $6 \mathrm{meV}$. This dispersionless mode contributes significant weight to the direction-averaged spectrum $S(Q, \omega)$ measured by powder INS [5,7]. However, the spectrum is dominated by a dispersive band, which exhibits a gap at the ordering wave vector $\mathbf{k}^{\prime}$. The dispersive band approaches this energy near the $Q_{l}=1$ zone boundary, roughly doubling the intensity there. Strong dispersion in all measured directions implies significant intercluster interactions. At $20 \mathrm{~K}$, the gap has collapsed, but both modes are still visible, although heavily broadened and somewhat softened in energy.

High-resolution data from cold neutron spectrometers reveal several novel features, Figs. 2(a)-2(d). The intense dispersive band actually consists of four separate modes, all dispersing according to the overall envelope seen on the lower-resolution thermal instrument, Fig. 1(b). These four modes are sharp and resolution limited. The value of the energy gap at $\mathbf{k}^{\prime}$ is $\Delta_{\mathrm{Cl}}^{\min }=1.99 \pm 0.01 \mathrm{meV}$. Careful searches below the gap revealed a very weak mode dispersing linearly away from the incommensurate Bragg peak, with no gap down to the experimentally accessible $0.5 \mathrm{meV}$. It is highly unusual that this Goldstone-like mode (GLM) [9] is much weaker than the gapped mode at $2 \mathrm{meV}$. In simple colinear systems, the neutron intensity can vanish if the fluctuations are along $\mathbf{Q}$. This cannot be the case for the ICM magnetic structure of CTC.

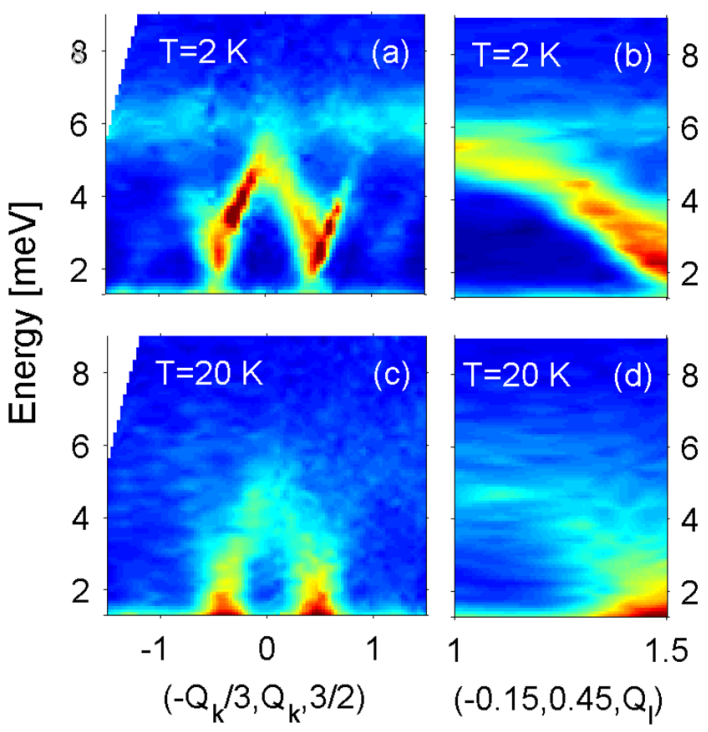

FIG. 1 (color online). Map of the excitation spectrum of CTC compiled from 96 individual energy scans on IN8 at $2 \mathrm{~K}$ (a)-(b) and at $20 \mathrm{~K}(\mathrm{c})-(\mathrm{d})$.
Our diffraction work [6] shows that two magnetic structures can exist in CTC, with propagation vectors $\mathbf{k}^{\prime}=$ $(-0.150,0.422,1 / 2)$ and $\mathbf{k}=(0.150,0.422,1 / 2)$. In our sample, only the $\mathbf{k}^{\prime}$ propagation wave vector was present. Still, tetragonal symmetry implies two domains: $\mathbf{k}^{\prime}$ and 90 degrees rotated $\mathbf{k}_{\mathbf{9 0}}^{\prime}$. It was therefore necessary to distinguish which of the four modes in Fig. 2(b) belong to which domain. We discovered that a moderate field of $2 \mathrm{~T}$ favors the ordering vector parallel to the field, thus producing a monodomain sample. This was exploited on the TASP spectrometer using vertical and horizontal field cryomagnets to select the $\mathbf{k}^{\prime}$ and $\mathbf{k}_{\mathbf{9 0}}^{\prime}$ domains, respectively, Fig. 2(c). At $\mathbf{Q}=\mathbf{k}^{\prime}$, two out of four peaks disappear upon application of the field, while the remaining two gain a factor of 2 in intensity. Hence, each domain contributes two modes to the dispersive band. Interestingly, the energy gap of $2.5 \mathrm{meV}$ at the ordering vector $\mathbf{Q}=\mathbf{k}^{\prime}$ is higher than the gap $\Delta_{\mathrm{Cl}}^{\min }$ at $\mathbf{k}_{\mathbf{9 0}}^{\prime}$. The magnetic order has lower symmetry than the tetragonal crystal lattice, so below $T_{N}$ the crystallographically equivalent $\mathbf{k}^{\prime}$ and $\mathbf{k}_{\mathbf{9 0}}^{\prime}$ points differ magnetically, and a priori need not have the same dispersion. The fact that for a $\mathbf{k}^{\prime}$ single domain phase, the energy gap at the nonpopulated $\mathbf{Q}=\mathbf{k}_{\mathbf{9 0}}^{\prime}$ wave vector has a minimum probably signals the proximity of an instability towards a double-k magnetic structure. The gap increase at $\mathbf{k}^{\prime}$ can be seen as repulsive hybridization with the GLM.

Next, in Fig. 3 we present the excitation spectrum of CTB measured at IN8. A gapped band, dispersing in all

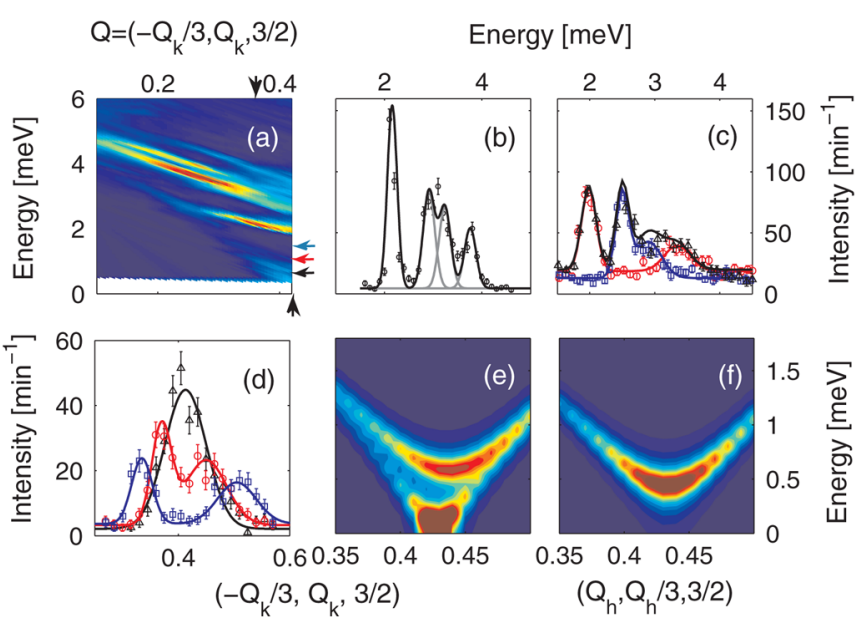

FIG. 2 (color online). High-resolution data on CTC at $1.5 \mathrm{~K}$. (a) $S(\mathbf{Q}, \omega)$ map compiled from 12 energy scans on IN14. (b) Energy scan from IN14 at $\mathbf{Q}=(-0.12,0.36,3 / 2)$ showing 4 resolution limited modes. (c) Energy scans at $\mathbf{k}^{\prime}$ from TASP in zero field (black triangles), $2 \mathrm{~T}$ parallel to $\mathbf{k}^{\prime}$ (blue squares) and $2 \mathrm{~T}$ perpendicular to $\mathbf{k}^{\prime}$ (red circles). Zero field intensities correspond to two half-populated domains and are multiplied by 2 . Blue and red lines are Gaussian fits. Their sum (black line) reproduces accurately the zero field intensity. (d) $\mathbf{Q}$ scans from IN14 of the low-energy mode at $0.75,1$, and $1.75 \mathrm{meV}$ (black triangles, red circles, blue squares). Lines are Gaussian fits. (ef) RPA calculation of the spectrum of CTC around $\mathbf{k}^{\prime}$ and $\mathbf{k}_{\mathbf{9 0}}^{\prime}$. 


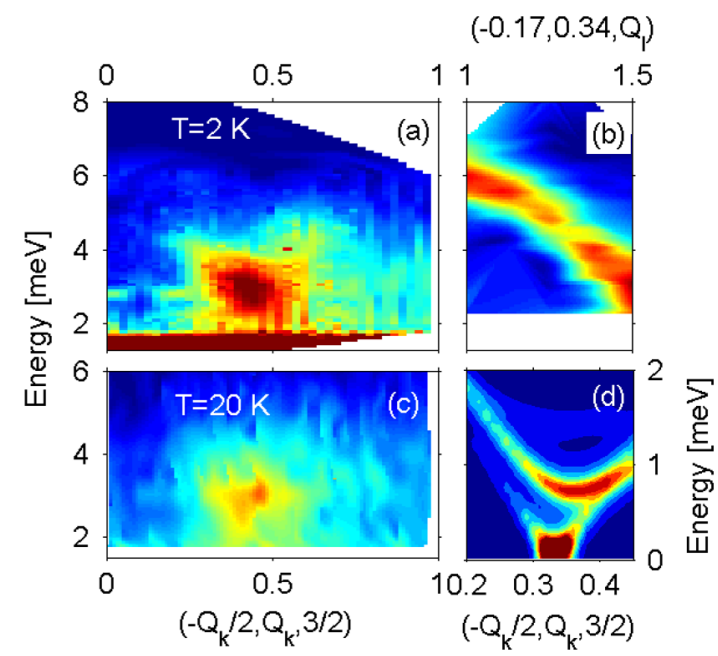

FIG. 3 (color online). Excitation spectrum of CTB obtained at IN8. (a)-(b) $S(\mathbf{Q}, \omega)$ maps at $2 \mathrm{~K}$ corresponding to Fig. 1(a) and 1(b). (c) Spectrum at $20 \mathrm{~K}$. The gapped excitation remains. (d) RPA-calculation of spectrum around $\mathbf{k}^{\prime}$ at $2 \mathrm{~K}$.

measured directions, similar to that of CTC, could be observed at $2 \mathrm{~K}$. The zone boundary energy $(6 \mathrm{meV})$ is similar to CTC, but the gap $\Delta_{\mathrm{Br}}^{\min }=2.6 \pm 0.1 \mathrm{meV}$ is higher. With the currently available sample size, highresolution measurements were not possible to confirm or exclude the presence of a weak GLM.

Finally, we compare in Fig. 4 the temperature dependence of the spectra at $\mathbf{k}^{\prime}$ for CTC and $\mathbf{k}^{\prime \prime}$ for CTB. In CTC, the gap at $\mathbf{k}^{\prime}$ closes at $T_{N}$, above which there is significant quasielastic scattering and only a weak inelastic component. The fits at $Q_{f}=(-0.075,0.225,3 / 2)$ and at $(0,0,1)$ show unusual temperature dependence. The intensity is almost $T$ independent with only a weak increase around $T_{N}$. The band energy decreases and damping increases as a function of temperature up to $T_{N}$, where both display a pronounced kink to much slower $T$ dependence. It is unlikely that the low- $T$ splitting in Fig. 2 would increase with temperature; hence, the widths can be interpreted as damping, except maybe above $25 \mathrm{~K}$ for $(0,0,1)$, where separation of optic and dispersive modes may contribute. At $(0,0,1)$ intensity and energy have only faint features at $T_{N}$. Together, this behavior is very unconventional for a 3D-coupled system, where the spin waves normally soften and dampen completely almost immediately above $T_{N}$. In lower-dimensional systems, damped spin-waves can persist well above $T_{N}$ due to a slowly decreasing correlation length [10,11]. Our findings suggest that significant correlations persist well above $T_{N}$ up to $k_{B} T \sim J$, or that the finite energy excitations above $T_{N}$ can be seen as heritage from the tetrahedral units. The situation is even more dramatic in CTB, where most of the spectral weight remains gapped beyond $T_{N}$, while only a weak signature of gap closing is observed. The different behaviors above $T_{N}$ in CTC and CTB derive from different ratios

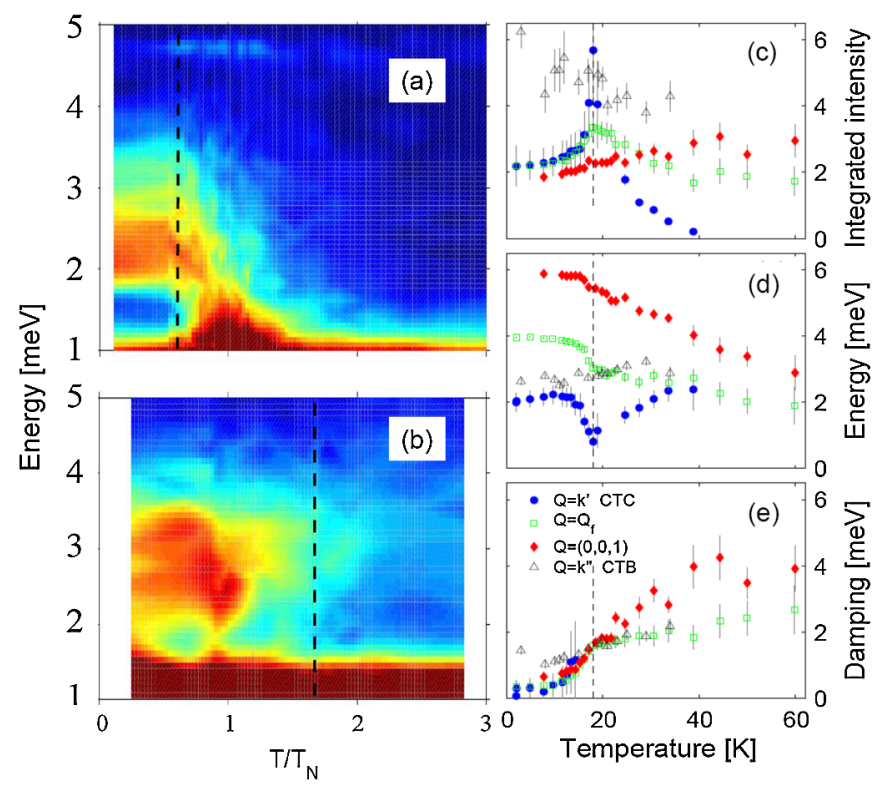

FIG. 4 (color online). (a)-(b) Spectrum at $\mathbf{k}^{\prime}$ and $\mathbf{k}^{\prime \prime}$ for CTC and CTB, respectively, measured on IN8. Dashed vertical lines indicate $k_{B} T=\Delta^{\mathrm{min}} / 2$. (c)-(e) Temperature dependence of integrated intensity, energy and damping (Lorentzian halfwidth) extracted by fitting energy scans at $\mathbf{k}^{\prime}, Q_{f}=$ $(-0.075,0.225,3 / 2)$ and $(0,0,1)$ in CTC and at $\mathbf{k}^{\prime \prime}$ in CTB. In CTC both $Q_{f}$, chosen to achieve optimal resolution focusing, and $(0,0,1)$ could be tracked to $60 \mathrm{~K}$. At $\mathbf{k}^{\prime}$ the most of intensity softens at $T_{N}$. The low- $T$ damping followed that of $Q_{f}$ and was fixed to the same $T$ dependence above $T_{N}$. Just above $T_{N}$ fits were unstable and have been omitted. In CTB, the smaller sample mass limited the analyzed range to $35 \mathrm{~K}$, and sample mosaicity prevented resolution focusing.

of ordering temperature versus minimum gap. In CTC, $k_{B} T_{N} \sim \Delta^{\mathrm{min}}$, meaning that once $T>T_{N}$, thermal fluctuations smear out the gap. In CTB, $k_{B} T_{N} \sim \Delta^{\mathrm{min}} / 3$, allowing the gapped excitation to exist in a temperature range above $T_{N}$ before fluctuations smear the spectrum.

To understand the excitation spectrum, a model was established, whose Hamiltonian includes Heisenberg, Dzyaloshinskii-Moriya (DM) and dipole-dipole interactions [12]. Parameters were self-consistently adjusted to reproduce the ordering vector $\mathbf{k}^{\prime}$, ordered moment, thermodynamic quantities and Raman spectra $[13,14]$. The tetrahedral clusters were diagonalized and a mean-field (MF) approximation was applied between clusters. The excitation spectra calculated within a random-phase approximation (RPA) and depicted in Figs. 2(e), 2(f), and 3(d), reproduce several salient features of our data. (i) At the ordering vector $\mathbf{k}^{\prime}$ there is both a GLM and a gapped mode. (ii) At $\mathbf{k}_{\mathbf{9 0}}^{\prime}$ there is only a gapped mode, with lower energy than at $\mathbf{k}^{\prime}$. (iii) CTB has a higher gap than CTC. (iv) There is a correspondence between the observed and calculated (not shown) high-temperature spectra for both compounds. There are, however, also important discrepancies between experiment and theory. The calculated 
gaps are almost 4 times smaller than observed, and the intensity of the calculated GLM is stronger than the gapped mode, contrary to experiment. This is surprising because MF/RPA theory should work well in the limit of weakly coupled tetrahedra and in the limit of well connected tetrahedra, where classical 3D correlations normally would dominate. To our understanding, any other conventional semiclassical theory for excitations of an ordered state will yield a similarly strong GLM and therefore not explain our findings.

We discuss three possible origins of these discrepancies. (i) Sample imperfections could pin the ordered structure, potentially shifting excitation energies. However, careful bulk measurements show no indications of magnetic defects. Furthermore, only the $\mathbf{k}^{\prime}$ domain is seen in our CTC sample, indicating good sample quality. It is further unlikely that imperfections could reduce the spectral weight of the GLM and raise the energy of the gapped mode without broadening Bragg peaks and excitations, all of which are resolution limited. (ii) It is possible to remedy the energy scale by introducing large DM interactions (or other anisotropic terms) in the Hamiltonian. However, this does not fix the relative intensities. (iii) The Hamiltonianparameter fits prescribe coupling along the $c$ axis and $a b$ planes comparable to the intracluster interactions [12], which we conclude lead to intercluster quantum fluctuations not contained in the MF/RPA.

Quantum fluctuations may renormalize excitation energies and intensities [15] and angles between the spins in an ICM structure [16]. Since the MF-Hamiltonian was tuned to match the ICM structure, the true Hamiltonian might have slightly different parameters and could yield slightly different renormalized energies. However, this would still give an intense GLM within MF/RPA.

When localized magnetic singlet units are coupled increasingly strongly into a 3D lattice, there may be a quantum phase transition to an ordered state, where the fundamental excitations change from singlet-triplet excitations to spin waves. In the coupled-dimer material $\mathrm{TlCuCl}_{3}$, the pressure-induced long-range order hosts Goldstone-like and gapped transverse spin-waves coexisting with a longitudinal mode [17], described in a theory coupling individual dimer states using a bond-operator formalism. However, in $\mathrm{TlCuCl}_{3}$ the excitation spectrum behaves in the usual manner where the low-energy GLM is more intense than the gapped modes.
To conclude, we have in $\mathrm{Cu}_{2} \mathrm{Te}_{2} \mathrm{O}_{5} X_{2}(X=\mathrm{Cl}, \mathrm{Br})$ discovered a new situation where an ordered magnetic structure of coupled clusters is accompanied by a very weak GLM (in CTC) and an intense gapped excitation (in both CTC and CTB). In addition to this discovery, we provide a full experimental description of the excitation spectra, and report an MF/RPA theory that accounts qualitatively for several features except the unusual intensity ratio and absolute gap value. We argue that this behavior is not limited to the specific materials CTC and CTB, but represent a new general type of scenario caused by intercluster quantum effects. We therefore call for efforts to develop a new theoretical paradigm capable of bridging the gap between local quantized clusters and the semiclassical excitations accompanying conventional long-range order. The first step in this challenge is to identify a minimal model (dimensionality, type of magnetic order, etc.) that captures the scenario.

We acknowledge F. Mila, M. Elhajal, Ch. Ruegg, and B. Normand for valuable input. The work was partially performed at SINQ, PSI, Switzerland and was supported by the Swiss NSF and the NCCR MaNEP.

*krunoslav.prsa@psi.ch

[1] B. Bleaney and K. Bowers, Proc. R. Soc. A 214, 451 (1952).

[2] M. Johnsson et al., Chem. Mater. 12, 2853 (2000).

[3] M. Prester et al., Phys. Rev. B 69, 180401(R) (2004).

[4] O. Zaharko et al., Phys. Rev. Lett. 93, 217206 (2004).

[5] O. Zaharko et al., Fiz. Nizk. Temp. 31, 1068 (2005).

[6] O. Zaharko et al., Phys. Rev. B 73, 064422 (2006).

[7] S. Crowe et al., Phys. Rev. B 71, 224430 (2005).

[8] K.-Y. Choi et al., Phys. Rev. B 79, 024416 (2009).

[9] The magnetic Hamiltonian of this system consists not only of Heisenberg exchange, but also of anisotropic terms. Hence, there is no symmetry requirement for the Goldstone mode and in the text the low-energy mode is referred to as the Goldstone-like mode.

[10] H. M. Rønnow et al., Phys. Rev. Lett. 82, 3152 (1999).

[11] H. M. Rønnow et al., Phys. Rev. Lett. 87, 037202 (2001).

[12] J. Jensen, Phys. Rev. B 79, 014406 (2009).

[13] C. Gros et al., Phys. Rev. B 67, 174405 (2003).

[14] J. Jensen et al., Europhys. Lett. 64, 689 (2003).

[15] N. B. Christensen et al., Proc. Natl. Acad. Sci. U.S.A. 104, 15264 (2007).

[16] M. Enderle et al., Europhys. Lett. 70, 237 (2005).

[17] Ch. Rüegg et al., Phys. Rev. Lett. 100, 205701 (2008). 